\title{
Modelo de desarrollo del conocimiento matemático desde el Saber y Hacer del pueblo mapuche
}

\section{Model of the development of mathematical knowledge from the Knowing and Doing of the Mapuche people}

\author{
Anahí Huencho ${ }^{1}$ \\ Eugenio Chandía ${ }^{2}$ \\ Francisco Rojas ${ }^{3}$ \\ Guillermo Williamson ${ }^{4}$
}

\begin{abstract}
Resumen: Dos siglos de escuelas monoculturales en Chile repercuten en una enseñanza de las matemáticas estáticas y conductistas a la población en general, aun cuando lo matemático se sitúa histórica y culturalmente en lógicas de procedimientos orales y/o prácticos en diferentes grupos socioculturales invisibles a la escuela. Así, esta investigación busca interpretar y caracterizar el desarrollo del conocimiento matemático del mapuche desde prácticas situadas de y en comunidades, con el anhelo de proyectarlas al sistema educativo formal y así corroer el modelo establecido en Educación Matemática chilena, dando evidencia de un modelo de enseñanza y aprendizaje de la matemática propio del pueblo mapuche. A través de un análisis de carácter mixto,
\end{abstract}

Fecha de recepción: 02 de diciembre de 2019. Fecha de aceptación: 11 de febrero de 2021.

1 Facultad de Educación, Universidad Católica de Temuco, Temuco, Chile. ahuencho@uctcl, orcid. org/0000-0001-6114-5332

2 Facultad de Educación, Universidad de Concepción, Concepción, Chile. echandia@udec.cl, orcid. org/0000-0003-2489-1226

3 Facultad de Educación, Pontificia Universidad Católica de Chile, Santiago, Chile. frojass@uc.cl, orcid. org/0000-0002-0328-8156

${ }^{4}$ Facultad de Educación, Universidad de la Frontera, Temuco, Chile. guillermo.williamson@ufrontera.cl, orcid.org/0000-0001-5313-342X 
utilizando el Método Comparativo Constante y el ACL sobre entrevistas en profundidad con foco etnográfico en el marco de la Teoría Fundamentada, se constituye un Modelo Condicional/Consecuencial que caracteriza el desarrollo del conocimiento matemático mapuche desde dos focos: el Saber, centrado en la memoria oral transmitida en lengua mapuche (mapudungun) al resolver problemas matemáticos contextuales y el Hacer, centrado en elementos matemáticos implícitos en la acción desarrollada.

Palabras claves: modelo educativo cultural, conocimiento matemático, matemática mapuche, práctica cultural situada.

\begin{abstract}
Two centuries of monocultural schools in Chile have an impact on the teaching of static and behavioral mathematics to the general population, even though the mathematics is historically and culturally situated in the logic of oral and / or practical procedures in different sociocultural groups invisible to the school. Thus, this research seeks to interpret and characterize the development of mathematical knowledge of the Mapuche from practices located in and from communities, with the desire to project them to the formal educational system and thus corrode the model established in Chilean Mathematical Education, giving evidence of a model of teaching and learning of mathematics typical of the Mapuche people. Through a mixed character analysis, using the Constant Comparative Method and the Analysis of Latent Classes about in-depth interviews with an ethnographic focus in the framework of the Grounded Theory, a Conditional / Consequential Model that characterizes the development of mathematical knowledge is constituted Mapuche from two focuses: Knowledge, centered on the oral memory transmitted in the Mapuche language (mapudungun) when solving contextual mathematical problems and the Do, focusing on mathematical elements implicit in the action developed.
\end{abstract}

Keywords: cultural educative model, mathematical knowledge, mapuche mathematics, situated cultural practice. 


\section{ANTECEDENTES}

Los procesos de escolarización de los pueblos originarios en Chile, y en particular del pueblo mapuche, se inician en el siglo XVIII y XIX, cuando diferentes credos religiosos crearon escuelas monoculturales basadas en la evangelización y castellanización, dejando ausentes de dichos procesos los saberes educativos de las distintas culturas (Flores y Azócar, 2006; Hanisch, 1974). Luego de dos siglos, la enseñanza monocultural transcendió en todos los niveles del sistema educativo chileno (Arias-Ortega et al., 2018; OCDE, 2009; Del Pino y Ferrada, 2019; Luna et al., 2018; Ortiz-Velosa y Arias-Ortega, 2019) perpetuando la cultura y los paradigmas de la enseñanza occidental, estableciéndolos como los únicos modelos válidos de enseñanza y aprendizaje (Ortiz-Velosa y Arias-Ortega, 2019; Quintriqueo et al., 2015). Entre las causas de la perpetuidad del modelo monocultural se puede mencionar que los educadores, de cualquier nivel educativo, desconocen la sociedad y cultura donde están inmersos sus estudiantes, tanto por el confort del modelo monocultural, como también por la falta de categorías de conocimiento y habilidades posibles de ser incorporadas en el sistema educativo en todas sus dimensiones construidas de manera conjunta entre investigadores-educadores y comunidades indígenas chilenas (Luna et al., 2018; Ortiz-Velosa y Arias-Ortega, 2019; Quintriqueo et al., 2015). Esto ha traído como consecuencia funesta, el abandono progresivo del conocimiento de la cultura de los pueblos originarios en Chile (Noggler, 1982; Quintriqueo, 2010), y por ende su olvido y desaparición.

Lo anterior pone urgencia a la identificación del conocimiento, habilidades e interpretación de los modelos de formación inherentes de los pueblos originarios de Chile mediante procesos metodológicos rigurosos que incluyan a las comunidades como parte integral de las investigaciones (Peña-Cortés et al., 2019). Para esto, se hace necesario comprender que un modelo se caracteriza como un sistema de interpretaciones y representaciones de situaciones específicas para objetivos establecidos, y que para lograr tales interpretaciones se debe usar un marco conceptual cognitivo que permita dar significado a lo percibido logrando representar el fenómeno. Este marco actúa como filtro, para seleccionar organizar, transformar o inferir patrones y regularidades bajo la superficie de lo que se quiere modelar (Lesh y Harel, 2003).

Por tanto, cualquier investigación que pretenda observar los modelos educativos del pueblo mapuche, debe reconocer que los resultados de su investigación podrían estar sesgados por la educación monocultural occidental que 
han recibido los integrantes de las comunidades indígenas (Luna et al., 2018; Quintriqueo et al., 2015) o bien por quien trata de interpretarlos. Por ejemplo, Alarcón y colegas (2018) interpretan que la formación del pueblo mapuche considera cuestiones político-contingentes como pivotes para el aprendizaje de niños y niñas, lo que lleva a tensionar los modelos tradicionales de enseñanza con las actuales necesidades de cada comunidad. Pese a esto, los kimche (personas sabias) conservan una sólida identidad sociocultural asociada a sus costumbres y los conocimientos parentales (Quintriqueo et al., 2015), resistiendo la incorporación de elementos de otras culturas (Alarcón et al., 2018).

En Chile, en la década de 1990, se crea el Programa de Educación Intercultural Bilingüe (PEIB), con el objetivo de nivelar los resultados educativos de estudiantes indígenas a los estándares de calidad nacional. Y en el año 2009, se establecen los objetivos de aprendizaje para la creación del Sector Lengua Indígena (SLI) en Educación Básica, con el objetivo de fortalecer los conocimientos culturales y lingüísticos de cuatro pueblos originarios que mantienen vigente su lengua vernácula: aymara, mapuche, quechua y rapa nui. Se ha ido conformando un proceso de institucionalización de la PEIB en el Estado que, pleno de contradicciones, avances y retrocesos, ha permitido ir posicionándola en el marco del sistema educacional, de sus políticas y programas (Williamson, 2012), aunque con carencias en transversalidad curricular, participación y adecuación cultural. Pese a estas iniciativas, los resultados de investigación muestran un bajo impacto en el quiebre del modelo monocultural en el aula escolar (Peña-Cortés et al., 2019). Aún más, los mismos niños y niñas mapuches expuestos a estas políticas educativas reconocen elementos que no pertenecen a su cultura (Peña-Cortés et al., 2019), particularmente en relación con los modelos de transmisión de conocimiento. Esto se acentúa en educación matemática, ya que las políticas educativas evidencian un carácter compensatorio y no igualitario (Huencho et al., 2017), sin mostrar evidencia de procesos matemáticos concretos asociados al pueblo mapuche (Huencho, 2015; S. Salas et al., 2015). Esto hace poco atractivo el uso de los recursos educativos generados desde cada política educativa por parte de los docentes (Ibañez, 2010), quienes reconocen la necesidad de modelos de enseñanza específicos a las comunidades donde están insertos sus estudiantes, así como también reconocen su carencia para desarrollar en el aula el conocimiento matemático inherente a las prácticas del mapuche.

Como consecuencia directa del bajo impacto de las políticas educativas y de la carencia de recursos didácticos y conocimientos, los estudiantes indígenas resultan ser mal preparados tanto en el modelo monocultural como en el 
intercultural (Canales y Webb, 2018; Undurraga, 2014). Se obtienen, por ejemplo, magros resultados en evaluaciones nacionales estandarizadas como el SIMCE ${ }^{5}$ o la PSU6 (Ortiz-Velosa y Arias-Ortega, 2019).

De lo anterior, esta investigación busca interpretar y caracterizar el desarrollo del conocimiento matemático del mapuche desde prácticas situadas de y en comunidades, con el anhelo de proyectarlas al sistema educativo formal y así corroer el modelo monocultural del sistema educativo chileno en el área de la Educación Matemática, dando evidencia de un modelo de enseñanza y aprendizaje de la matemática propio del pueblo mapuche. Así, el objetivo de este estudio es construir un Modelo Condicional/Consecuencial que caracterize el desarrollo del conocimiento matemático mapuche que emerge desde las prácticas y los relatos de las prácticas socioculturales propias del pueblo.

\section{DESARROLLO DE CONOCIMIENTO MATEMÁTICO SITUADO}

Los seres humanos, como agentes activos de su desarrollo cognitivo, construyen marcos contextuales y sistemas lógicos de acción, lo que da pie al desarrollo de un conocimiento sociocultural propio (D'Ambrosio, 2007; Fyhn et al., 2016). Los sujetos, por medio de prácticas culturales tradicionales, interactúan con herramientas concretas y abstractas, complejas de evidenciar para un externo a la comunidad. El desarrollo de conocimiento sociocultural implica la transformación de las formas de percibir y comprender el mundo, a través de la interacción entre sujetos en un medio común (Rogoff, 2003). La producción científica en educación matemática es un campo en el que se disputan opciones conceptuales, metodológicas y políticas frente a la relación intercultural de saberes, que entre otros temas, atiende a la reinvención de prácticas de insubordinación creativa o subversión responsable (D'Ambrosio y Lopes, 2015) frente a la enseñanza monocultural, al curriculum que privilegia el enfoque cultural dominante y una pedagogía que subordina la diversidad a la homogeneidad cultural, lingüística y territorial del sistema educacional.

El desarrollo del conocimiento cultural mapuche se enmarca en la historia local, el territorio y el parentesco, los cuales configuran la educación familiar en el proceso de formarse como mapuche (Quintriqueo et al., 2015; Quintriqueo y Torres,

\footnotetext{
${ }^{5}$ Sistema Nacional de Evaluación de resultados de aprendizaje del Ministerio de Educación de Chile.

${ }^{6}$ Prueba de Selección Universitaria de Chile.
} 
2013). La interacción entre el que enseña y el que aprende con foco en cultivar y construir conocimiento mapuche, se sustenta en diversas prácticas culturales tradicionales donde se reflejan elementos conceptuales, procedimentales y valórico-actitudinales (Quilaqueo et al., 2016; Quilaqueo y San Martín, 2008).

Desde una visión dinámica y constructivista de las matemáticas (Felbrich et al., 2012; Kaiser y Maaß, 2007), las prácticas matemáticas tienen significado en sí mismas y se desarrollan dentro de un contexto en el que lo matemático se sitúa histórica y culturalmente en lógicas de procedimientos orales y/o prácticos en diferentes grupos socioculturales (Bishop, 1991; Sierpinska y Lerman, 1996). Así, la matemática que un observador pueda relacionar con un objeto, proceso o explicación, requiere de una interpretación situada para identificarla en las prácticas cotidianas de los diversos grupos socioculturales (Albertí, 2007). En el contexto mapuche, se ha podido identificar información matemática desde su composición lingüística y contexto de uso en el ámbito de los numerales y cuantificadores de tiempo, espacio, longitud y capacidad (Catrileo, 1985; Peña-Rincón y Hueitra-Santibañez, 2016; A. Salas, 1980). En el caso de los pueblos ágrafos, como ha sido el caso mapuche, la memoria oral es la clave que sustenta su desarrollo conceptual (Ong, 1987), lo que matemáticamente restringe las variedades de representaciones posibles.

\section{MODELOS PARA LA COMPRENSIÓN DE FENÓMENOS}

Los modelos se han usado tanto para describir la actividad matemática general o específica (Kuzniak, 2011; Van Hiele, 1959; Vygotsky, 1978), como para explicar, representar y comprender el proceso de enseñanza y aprendizaje observado en cualquier nivel educacional (Blömeke et al., 2012; Brousseau, 1986; McDonald et al., 2014; Shulman, 1987; Turner y Rowland, 2011).

Barbé y colegas (2005) plantean que el uso de modelos queda establecido por la realidad que el investigador quiere abordar. Así, cualquier modelo constituiría una propuesta metodológica de aproximación a la realidad diseñada por el investigador. Por tanto, el uso demostraría la comprensión de los componentes, patrones y relaciones de un fenómeno en relación con el objetivo de investigación, lo que equivaldría a un análisis del fenómeno; por ser planteado por un sujeto con intereses propios y marcos de disposiciones cognitivas y afectivas propias de su competencia profesional debería ser constantemente cuestionado y confrontado empíricamente (Blömeke et al., 2012; Chevallard y Bosch, 2014). 
De lo anterior, se puede establecer que los modelos son usados por las teorías principalmente para salir de su espacio conceptual al espacio empírico de desarrollo; los investigadores continuamente lo realizan al tratar de explicar, comprender o relacionar fenómenos con base en teorías pre-existentes o con el objetivo de crear nuevas, por ejemplo: al establecer modelos en la actividad matemática, comprender los modelos de los estudiantes, o representar el proceso de interacción en ambientes de aprendizaje (Lesh et al., 2013). Debido a esto, se puede considerar que los modelos son principalmente usados para compartir con otros un fenómeno. Así, el modelado es inherentemente una empresa social y están involucradas formas significativas de generalización y transferibilidad (Lesh et al., 2013).

Dentro del fenómeno cultural, también se observan modelos que tratan de abordarlo, interpretando y explicando la cultura de una comunidad, o bien, su transferencia. En este último caso, los modelos que tratan de explicar la transferencia cultural, se posicionan en marcos conceptuales sociales de aprendizaje (Miller et al., 1966).

Entre los modelos de transferencia cultural, destaca el propuesto por Chau (1990), el cual consta de cuatro cuadrantes definidos por dos ejes. El eje horizontal, representa el continuo valor ideológico del etnocentrismo y el pluralismo, y el eje vertical define los objetivos de la intervención, organizados en un continuo, desde el cambio individual al cambio socioestructural. Así, el modelo no solo integra el conocimiento cultural a otro espacio de conocimiento, si no que distribuye la "transferencia o métodos de enseñanza" entre los objetivos micro y macrosociales que se quieren alcanzar con la combinación de los diferentes tipos de conocimiento. Lo anterior, permite establecer que los modelos culturales de enseñanza y aprendizaje deberían explorarse en las intersecciones de la cognición del instructor, las prácticas de enseñanza y los entornos de instrucción. Esta visión relacional sugiere que la implementación de los modelos culturales de enseñanza y aprendizaje implican necesariamente una transformación del entorno y las formas en que esos entornos se perciben como un apoyo o restricción de ciertas acciones. De hecho, es difícil imaginar la evolución de estos sin la coevolución de los entornos en los que se construyen y se representan (Ferrare y Hora, 2014).

Los modelos explicativos de la cultura de una comunidad, también son diversos dada las diferentes definiciones de cultura, así como de investigadores que la tratan de explicar. Sin embargo, se distribuyen en una dimensión que tiene en un extremo teorías de aprendizaje y enseñanza y en el otro extremo 
datos empíricos de las comunidades, como lo explican Ferrare y Hora en su revisión de este tipo de modelos (2014). Los modelos teóricos culturales se han usado para comprender como individuos internalizan, organizan y promueven el conocimiento cultural. Por otra parte, los modelos empíricos se han usado para describir como las personas, eventos y objetos se ajustan en conjunto (Ferrare y Hora, 2014). De esta forma los modelos culturales consisten en información compartida que es internalizada a través de patrones de socialización entre y en grupos. En esta línea, antropólogos cognitivos definen los modelos culturales como teorías simplificadas sobre relaciones entre las personas, las prácticas y los eventos que se desarrollan a través de la activación repetida de las redes neuronales en relación con tareas y situaciones específicas (Ferrare y Hora, 2014).

De lo anterior, los modelos que tratan de explicar una cultura o la transferencia de ella son un ejemplo empírico y específico de la identificación de condiciones y consecuencias a niveles micro y macro de acciones e interacciones de los sujetos que la componen para el establecimiento de un núcleo central como representación y comprensión de los mismos en su cultura (Restrepo-Ochoa, 2013; Strauss y Corbin, 2002).

\section{METODOLOGÍA}

Dado el objetivo del estudio se considera un diseño cualitativo observacional. La técnica principal corresponde a entrevistas en profundidad con foco etnográfico dentro del marco de la Teoría Fundamentada. Se cumplieron los protocolos éticos exigidos por las universidades para esta modalidad de investigación, así como se respetaron los protocolos de conversación con las comunidades y sujetos participantes. El análisis de los datos fue de carácter mixto, utilizando el Método Comparativo Constante (Strauss y Corbin, 2002) y el ACL (Magidson y Vermunt, 2004).

En la investigación participaron 51 personas pertenecientes al pueblo mapuche (28 mujeres y 23 hombres), con edades entre 30 y 94 años. De ellos, 35 tienen como lengua materna el mapudungun (lengua mapuche), y el resto son hablantes o entienden la lengua. Se trabajó con las comunidades mapuche de Peuman Mapu, Lladquihue Norte y Lladquihue Sur, en las ciudades de Lautaro y Temuco, Región de la Araucanía, Chile. En cada comunidad, un integrante de confianza de esta seleccionó a sus representantes y guió la gestión de cada entrevista. 
La entrevista en profundidad con enfoque etnográfico contempló lo siguiente: a) Historia de vida; b) Actividad con matemática implícita que realizaban o realizan; y c) Quién le enseñó y cómo lo aprendió. Las entrevistas se realizaron en el hogar de cada participante, en un transcurso de 10 meses. Cada entrevista fue videograbada, con un tiempo promedio de 2,5 horas de duración. La flexibilidad de la entrevista fue necesaria para adaptarse al perfil de los informantes y contextos en que ocurría el trabajo de campo.

Dentro de la Teoría Fundamentada, el procedimiento de análisis utilizado puede ser de origen cualitativo y/o cuantitativo, mientras no se pierda el foco dentro de la Teoría (Verd y Lozares, 2016). Para esta investigación se utilizó, por una parte, el Método Comparativo Constante con tal de organizar la información y establecer relaciones sustantivas y, por otra, el Análisis de Clases Latentes (ACL) (Magidson y Vermunt, 2004) para determinar un modelo que sea condicionado a la información que entrega cada individuo, tomando en cuenta la diversidad de experiencias de los participantes. Haciendo uso del registro de audio, se procedió por medio de ATLAS.ti a indexar los segmentos de las 11 primeras entrevistas para luego proceder a codificarlas con carga teórica, estableciendo así los indicadores de investigación. El rango escogido respondió a la necesidad de cubrir un margen aceptable de saturación que según Marshall et al., (2013) disminuye en $75 \%$ la aparición de nuevos indicadores cuando el rango de entrevistas va de 7 a 12.

Con un marco significativo de indicadores, se prosiguió a establecer el peso conceptual de las intervenciones según la cantidad de características mencionadas en las entrevistas asociadas a un concepto o categoría, variando entre (1) una, (2) dos y (3) tres o más características. Finalmente se procede a indexar y establecer el peso conceptual de las restantes entrevistas, estableciendo relaciones mediante herramientas para importar códigos, comunes vecinos, co-ocurrencia o tablas de co-ocurrencia, y así determinar grupos de indicadores denominados "dimensiones" y conceptualizar sus relaciones internas y externas (Miles et al., 2014).

Codificadas las entrevistas se procedió a establecer un Modelo Condicional/ Consecuencial (Strauss y Corbin, 2002), usando el ACL para identificar asociaciones entre participantes que comparten características o elementos similares (Magidson y Vermunt, 2004; Rost, 2004). El ACL realiza clasificaciones basado en probabilidades, siendo los participantes clasificados en un perfil según la probabilidad estimada de pertenecer a él. De esta forma, los indicadores pueden ser agrupados en función de la distribución de presencia o ausencia y sus 
correspondientes niveles de caracterización, generando relaciones entre los participantes y, por ende, entre las clases latentes que los diferencian y los agrupan en perfiles. El análisis fue hecho con la función "poLCA" del programa R-project (Finch y French, 2015). El número de clases se determinó con base en el criterio de información Akaike y Bayesiana (AIC y BIC), considerando que el menor valor en ambos criterios indica un modelo de clases latentes mejor ajustado (Rost, 2004).

Para caracterizar el Modelo y sus clases latentes, se procedió a seleccionar indicadores representativos de cada clase latente según el grupo de participantes que la componen en dos formas diferentes: a) indicadores con probabilidad condicional de 0.5 o más, y b) comparación entre las probabilidades condicionales de cada indicador de cada clase latente. Apartados los indicadores, se evalúa la estructura lógica que conceptualiza cada clase latente dentro del Modelo Condicional/Consecuencial. La información organizada se visualiza de forma compacta y accesible por medio de redes conceptuales/relacionales (Miles et al., 2014).

\section{RESULTADOS}

\subsection{ANÁLISIS DESCRIPTIVO}

Desde el análisis de las entrevistas, el Desarrollo del Conocimiento Matemático Mapuche (DCMM) se descompone analíticamente en dos dimensiones, el Conocimiento Matemático Mapuche (CMM) y el Desarrollo del Conocimiento Mapuche (DCM), ambos caracterizados por indicadores que desagregan sus respectivos componentes conceptuales (ver tabla 1 ). 
Tabla 1. Dimensiones de clasificación del DCMM

\begin{tabular}{|c|c|}
\hline Dimensiones & Indicadores \\
\hline $\begin{array}{l}\text { Conocimiento } \\
\text { Matemático } \\
\text { Mapuche (CMM) }\end{array}$ & $\begin{array}{l}\text { Actividades: involucran el conocimiento ancestral y matemático de forma } \\
\text { implícita en actividades cotidianas del mapuche. } \\
\text { - Cuidar animales (AANI): proporcionar alimento y espacio físico a } \\
\text { animales. } \\
\text { - Tejer (ATEJ): dividido en tejer a telar con lana de oveja o cestos con } \\
\text { fibra vegetal. } \\
\text { - Construir (ACON): relacionada con el uso de madera, fibra vegetal y } \\
\text { herramientas. } \\
\text { - Sembrar (ASEM): asociada a la semilla y su técnica de incorporación } \\
\text { en la tierra. } \\
\text { - Cocinar (ACOC): manipulación de víveres variados para proveer de } \\
\text { alimento. } \\
\text { Objetos matemáticos: descripción de objetos o elementos que se emplean } \\
\text { para determinar cierta estructura matemática en diferentes actividades. } \\
\text { - Longitud (OLON): unidades de medidas no estandarizados como } \\
\text { pasos, varas, etc. } \\
\text { - Tiempo (OTIE): unidades de tiempo asociados a la ubicación de } \\
\text { astros en el cielo. } \\
\text { - Capacidad (OCAP): capacidad volumétrica no convencional, cestos o } \\
\text { vasijas. } \\
\text { - Cuantificador (OCUA): representar elementos a través de nudos, } \\
\text { palos o marcas. } \\
\text { Acciones matemáticas: explicitan un conjunto de tareas propias de una } \\
\text { práctica matemática que proporcionan después de su ejecución } \\
\text { matemática. } \\
\text { - Medir (AcMED): aplicar un patrón de longitud, tiempo o capacidad, } \\
\text { que se repite de manera uniforme y constante, obteniendo una } \\
\text { cuantificación oral de la medida. } \\
\text { - Contar (AcCON): aplicar una estrategia de enumeración que permita } \\
\text { obtener una cuantificación, comunicándola por medio de un sistema } \\
\text { numérico propio y oral. } \\
\text { - Representar (AcREP): aplicar un medio de representación de } \\
\text { cantidades, que permita visualizar la descomposición de la cantidad } \\
\text { en unidades y la base del sistema numérico. } \\
\text { Comparar (AcCOM): aplicar una relación aditiva (diferencia) y } \\
\text { multiplicativa (razón) en el proceso de cuantificación, comunicando } \\
\text { oralmente o por medio de representaciones. }\end{array}$ \\
\hline
\end{tabular}




\begin{tabular}{|c|c|}
\hline $\begin{array}{l}\text { Desarrollo del } \\
\text { Conocimiento } \\
\text { Mapuche (DCM) }\end{array}$ & 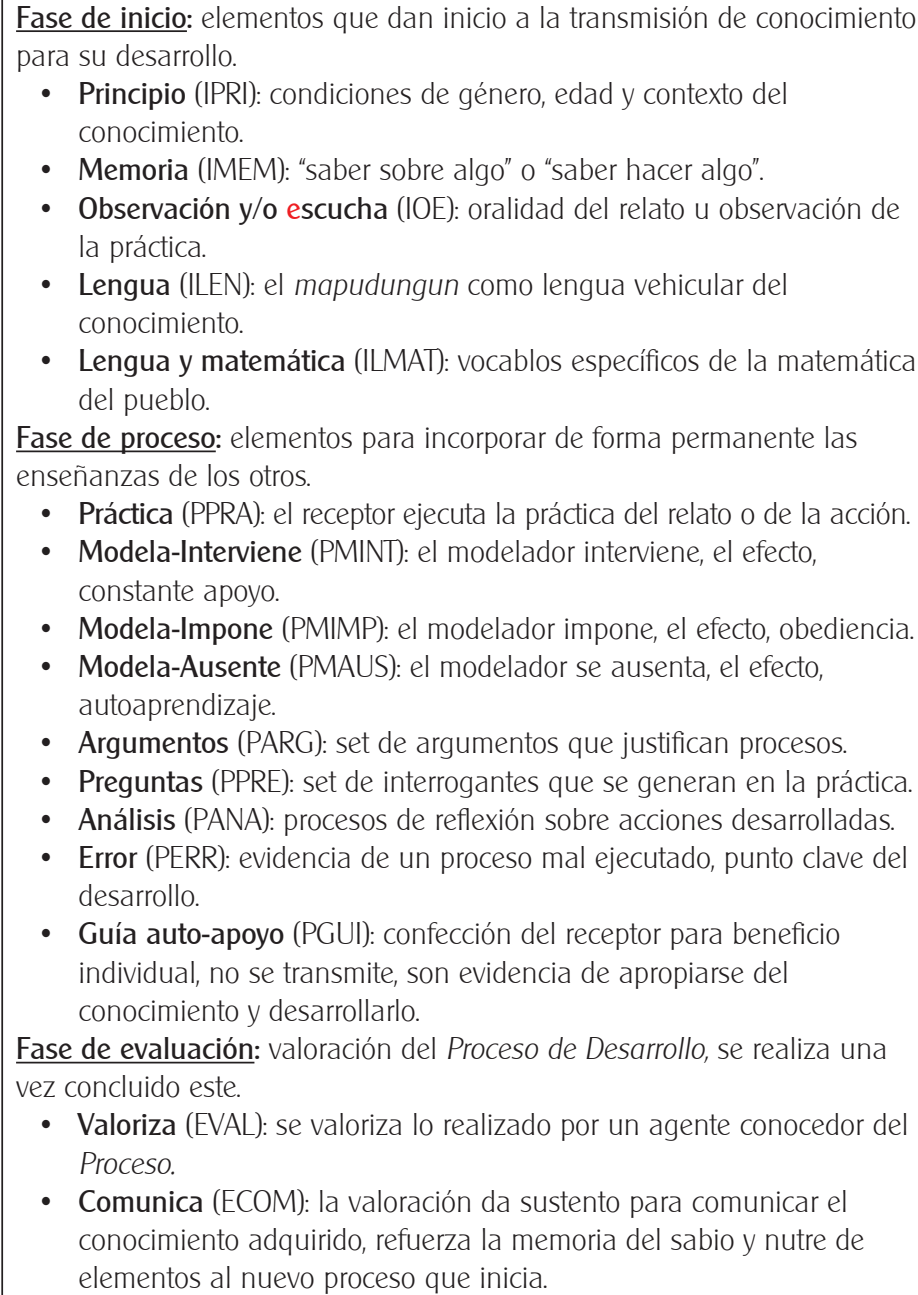 \\
\hline
\end{tabular}

Al determinar la frecuencia, se observa que aquellos indicadores asociados a CMM presentan una frecuencia absoluta acumulada menor a la mitad de la frecuencia absoluta acumulada de los indicadores asociados a DCM. Esto se debe a que cada entrevistado posee la habilidad para hacer o recordar información, que no necesariamente atiende a los indicadores emergentes para la dimensión CMM. En cambio, y como se observa en la figura 1, los indicadores 
asociados a la categoría DCM fueron observados en todas las entrevistas, independiente de la actividad de base, pero con claras diferencias en las rutas consideradas para el inicio, proceso y evaluación del desarrollo experimentado.

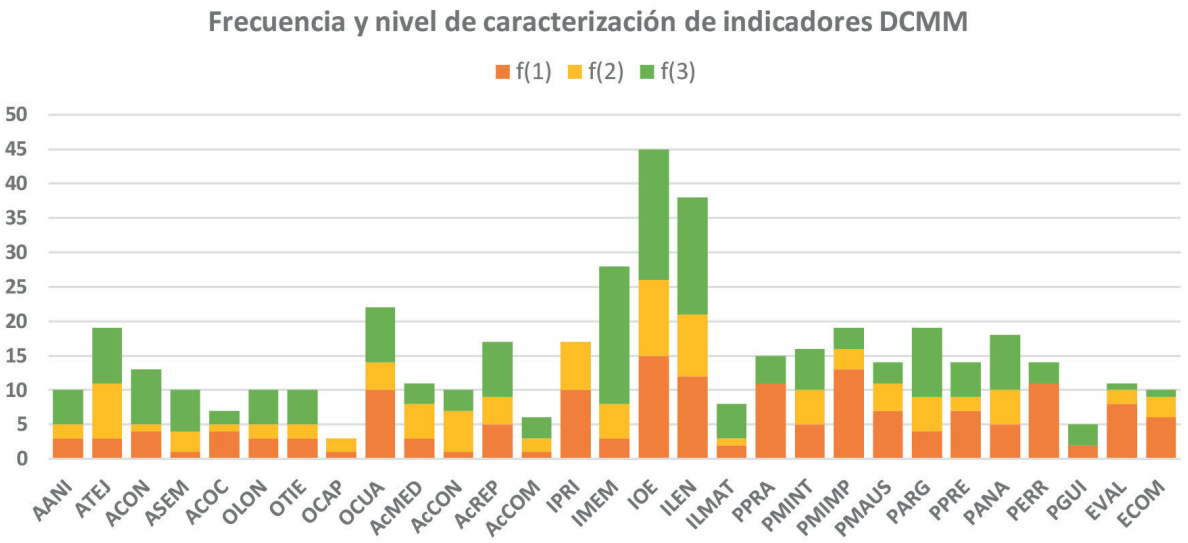

Figura 1. Frecuencia absoluta de indicadores DCMM.

Nota: $\mathrm{f(1)}$ una característica; $\mathrm{f(2)}$ dos características; f(3) tres o más características diferentes

De los 51 participantes, 45 mencionan la observación-escucha de una práctica o relato (IOE) como fundamental al desarrollar el conocimiento en el pueblo. Este indicador, IOE, es caracterizado de manera profunda por 19 de los 45 participantes. Por otra parte, el indicador OCAP es el que presenta menor frecuencia, siendo mencionado a nivel superficial por solo 3 entrevistados.

\subsection{ANÁLISIS RELACIONAL DENTRO DE CADA DIMENSIÓN}

\subsubsection{Conocimiento Matemático Mapuche (CMM)}

En la figura 2 se evidencian las relaciones entre las destrezas reflejadas a través de actividades cotidianas que poseen matemática implícita y la forma de explicitar estos elementos a través de objetos y acciones matemáticas emergentes. Así, desde las actividades que contienen objetos matemáticos como cuidar animales (AANI), tejer (ATEJ), construir (ACON), sembrar (ASEM) y cocinar (ACOC), 
se desprenden las acciones matemáticas involucradas como medir (AcMED), contar (AcCON), representar (AcREP) y comparar (AcCOM) en la manipulación conceptual de los objetos matemáticos de longitud (OLON), tiempo (OTIE), capacidad (OCAP), cuantificador (OCUA).

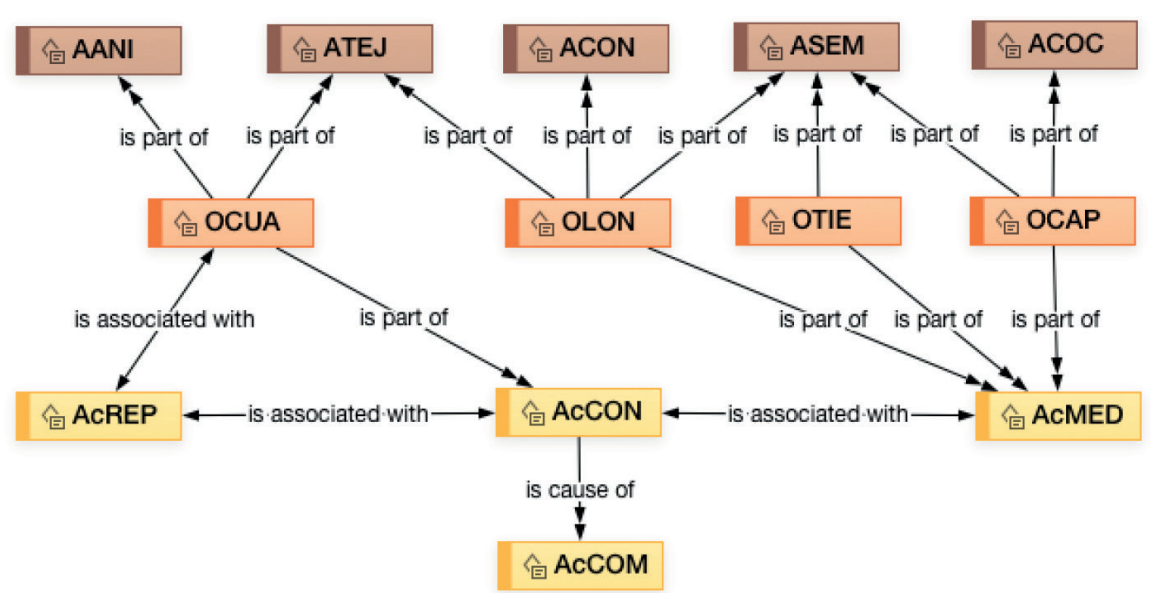

Figura 2. Relación de los indicadores dentro de la dimensión CMM.

De las actividades relativas al cuidado de animales (AANI) y a tejer (ATEJ), se desprende el uso de objetos cuantificadores (OCUA) por medio de la acción de representar (AcREP) asociado al conteo (AcCON) en lengua mapuche. Ahora, una cuestión interesante es que en las actividades de tejer, construir, sembrar y cocinar el proceso de medir, se observaron los objetos de longitud (OLON), tiempo (OTIE) y capacidad para cuantificar y dejar registro de esto (OCUA), principalmente por la acción de medir (AcMED). Esto se debe a que medir se asocia al proceso de contar, es decir cuantificar la medida, y este con el proceso de representar dada la necesidad de registrar la información cuantificada. Por último, la acción de comparar (AcCOM) se asocia a la acción de contar (AcCON) de los elementos, originando el objeto matemático de cuantificar (OCUA) en las actividades de construir, sembrar, y cocinar. Por ejemplo, la siguiente cita evidencia que, en la actividad de siembra, se mide (AcMED) la capacidad (OCAP) de los sacos cuantificándolos (OCUA + AcCON). Por otra parte, se observa la representación (AcREP) de estas cantidades (OCUA) en lana. 
Una siembra buena de lupino es la que da 40 sacos por 200 kilos de semilla, eso es para 1 hectárea (...), uno cosecha y guarda en sacos de 80 kilos cada uno. Si quiere más, puede sembrar hectárea y media, pero necesita 300 kilos de lupino, casi 4 sacos de esos. En un año bueno debiera darle 60 sacos de cosecha. (...) mi abuelo anotaba todo lo que había cosechado en lanas de diferentes colores con nudos y se lo pasaba a mi abuela. Ella repartía, cambiaba por otra cosa, el trueque, y cuidaba que alcanzara. [E23]

\subsubsection{Desarrollo del Conocimiento del Pueblo Mapuche (DCM)}

En las relaciones de los indicadores del DCM se caracterizan los elementos asociados a cada una de las fases de inicio, proceso y evaluación (figura 3). La fase de inicio del DCM enfatiza las condiciones y formas en las que el conocimiento mapuche se expresa. Se centra en la observación-escucha (IOE) que genera una persona con amplia capacidad de recordar hechos (IMEM) o replicar procesos apoyado del mapudungun. Así, desde el mapudungun (ILEN), se incorpora un conjunto de vocablos asociados a objetos y acciones matemáticas (ILMAT) dentro de una actividad que se inicia dependiendo de la edad, el género y la necesidad que impulsa la enseñanza (IPRI).

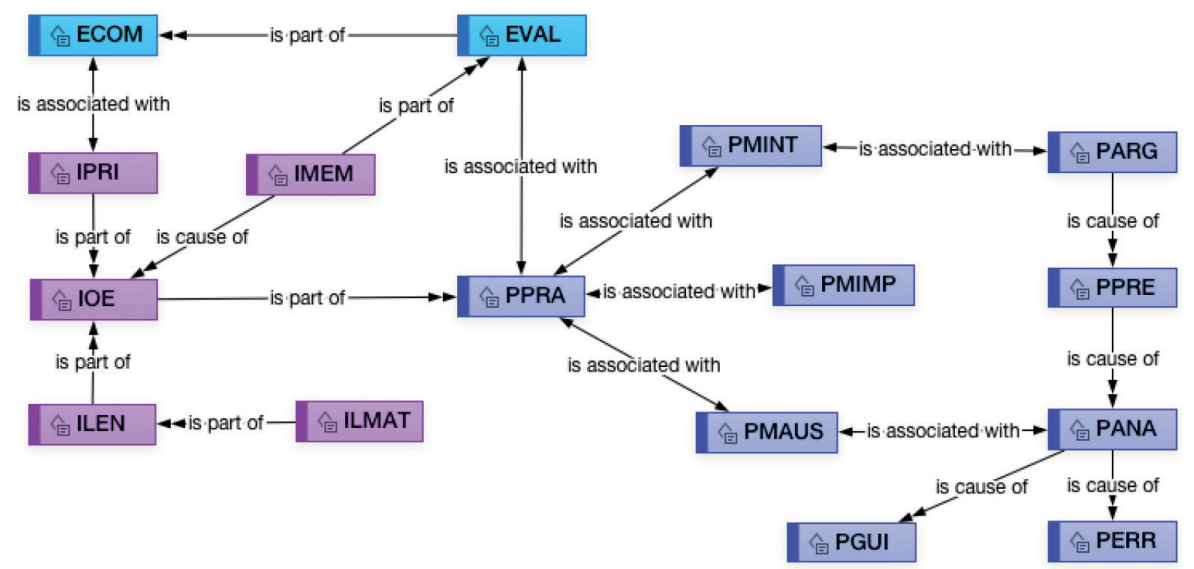

Figura 3. Relación de los indicadores dentro de la dimensión DCM. 
En la fase de proceso del DCM se observa que la principal forma de transferencia es el sujeto modelador. Él es quien, en sus formas de mediar el conocimiento y en sus respectivas consecuencias sobre el agente que inicia su adquisición y desarrollo, determina tres rutas de desenlace diferenciadas. En la primera, el agente que modela interviene (PMINT) en el proceso de reproducir la acción desde el error (PERR), analiza (PANA), cuestiona la práctica (PPRE), argumenta (PARG) y retroalimenta por medio de explicaciones. En la segunda, el modelador impone (PMIMP) reglas, formas, acciones, esperando la obediencia del ejecutor. La última ruta se genera desde un proceso autónomo en donde el modelador natural se ausenta (PMAUS) y la adquisición del conocimiento depende del error (PERR) y su auto-análisis (PANA). El proceso puede generar la confección de una guía de apoyo (PGUI) para el auto-modelamiento en futuras acciones. Finalmente, en la fase de evaluación del DCM, se valida (EVAL) y potencia conceptualmente el ciclo del desarrollo. Esto lo realiza un agente con amplio conocimiento en la materia, interno o externo al proceso, generando una comunicación que nutre el conocimiento y contribuye a iniciar nuevos ciclos de enseñanza (ECOM).

Mi papá me enseñó a construir kultrun [tambor mapuche], iuf! Las herramientas de antes no son como las de ahora, el papá me paso mi trozo de madera y me dijo que lo imitara, él me explicó cómo debía hacer, yo pensé y me hice mi propia ayuda memoria de todo lo que hice mal. Un día mi abuelo me alabó mi kultrun, ahora ya sabía hacer. [E18]

En la cita se evidencia que el entrevistado se sometió a un modelamiento (PMINT) que fue intervenido por los consejos y explicaciones del padre, quién le plantea que debe "imitar" (IOE). Luego se observa un proceso de reflexión de las acciones desarrolladas (PANA), considerando aquellas cuestiones mal ejecutadas (PERR), estableciendo por último una guía de autoayuda (PGUI). Por último, se observa la valorización del proceso de desarrollo por parte del abuelo (EVAL).

\subsection{Modelo Condicional/Consecuencial para DCMM}

En la última etapa de análisis y con el objetivo de levantar información para construir un Modelo Condicional/Consecuencial, se procedió a realizar un ACL para indagar en los patrones de respuesta y generar modelos acordes a las 
particularidades de los entrevistados identificando posibles perfiles comunes entre ellos. De esta manera, se estimaron 2 modelos con dos y tres clases latentes, de los cuales el de dos clases latentes obtuvo el mejor ajuste $(\mathrm{AIC}=2451.98$; $\mathrm{BIC}=2790.05)$. Así, se procedió a conceptualizar el DCMM desde el modelo con dos clases latentes. La tabla 2 deja en evidencia las probabilidades condicionales por cada indicador según su clase latente.

Tabla 2. Distribución de probabilidades por indicador condicionado a estar en $\mathrm{C} 1$ o C2 y por estar caracterizado por 1, 2 o 3 elementos.

\begin{tabular}{|c|c|c|c|c|c|c|c|c|c|}
\hline \multirow{2}{*}{\multicolumn{2}{|c|}{$\begin{array}{c}\text { Indicadores - I } \\
\text { Actividades }\end{array}$}} & & \multicolumn{4}{|c|}{$\begin{array}{c}\text { clase latente 1(C1) } \\
\text { DCMM desde el Saber }\end{array}$} & \multicolumn{3}{|c|}{$\begin{array}{c}\text { clase latente } 2 \text { (C2) } \\
\text { DCMM desde el } \\
\text { Hacer }\end{array}$} \\
\hline & & & & & & & & & \\
\hline \multirow{15}{*}{ 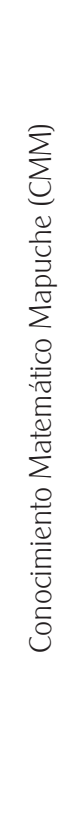 } & AANI & .17 & .00 & .08 & .08 & .15 & .08 & .03 & .05 \\
\hline & ATEJ & .08 & .00 & .08 & .00 & .41 & .08 & .18 & .15 \\
\hline & ACON & .00 & .00 & .00 & .00 & .26 & .10 & .03 & .13 \\
\hline & ASEM & .08 & .00 & .00 & .08 & .18 & .03 & .08 & .08 \\
\hline & ACOC & .00 & .00 & .00 & .00 & .15 & .10 & .03 & .03 \\
\hline & Objetos & & & & & & & & \\
\hline & OLON & .17 & .00 & .08 & .08 & .20 & .08 & .03 & .10 \\
\hline & OTIE & .25 & .00 & .00 & .25 & .18 & .08 & .05 & .05 \\
\hline & OCAP & .00 & .00 & .00 & .00 & .08 & .03 & .05 & .00 \\
\hline & OCUA & .70 & .06 & .05 & .59 & .21 & .09 & .09 & .03 \\
\hline & Acciones & & & & & & & & \\
\hline & AcMED & .33 & .08 & .25 & .00 & .18 & .05 & .05 & .08 \\
\hline & AcCON & .17 & .00 & .17 & .00 & .21 & .03 & .10 & .08 \\
\hline & AcREP & .75 & .08 & .08 & .59 & .21 & .10 & .08 & .03 \\
\hline & ACPCOM & .17 & .08 & .00 & .08 & .10 & .00 & .05 & .05 \\
\hline
\end{tabular}




\begin{tabular}{|c|c|c|c|c|c|c|c|c|}
\hline \multirow{19}{*}{ 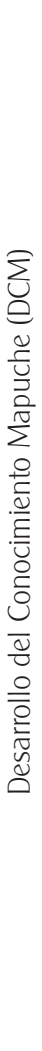 } & \multicolumn{8}{|l|}{ Inicio } \\
\hline & IPRI & .08 & .00 & .08 & .00 & .41 & .26 & .15 \\
\hline & IMEM & .50 & .05 & .13 & .31 & .28 & .13 & .13 \\
\hline & IOE & .83 & .00 & .00 & .83 & .90 & .38 & .28 \\
\hline & ILEN & 1.00 & .08 & .08 & .83 & .67 & .28 & .20 \\
\hline & ILMAT & .42 & .00 & .00 & .42 & .08 & .05 & .03 \\
\hline & \multicolumn{8}{|l|}{ Proceso } \\
\hline & PPRA & .17 & .17 & .00 & .00 & .33 & .23 & .00 \\
\hline & PMINT & .41 & .00 & .00 & .41 & .28 & .13 & .13 \\
\hline & PMIMP & .08 & .00 & .00 & .08 & .46 & .33 & .08 \\
\hline & PMAUS & .08 & .00 & .00 & .08 & .33 & .18 & .10 \\
\hline & PARG & .50 & .00 & .08 & .42 & .33 & .10 & .10 \\
\hline & PPRE & .59 & .08 & .17 & .33 & .18 & .15 & .00 \\
\hline & PANA & .33 & .00 & .08 & .25 & .36 & .13 & .10 \\
\hline & PERR & .33 & .25 & .00 & .08 & .26 & .21 & .00 \\
\hline & PGUI & .08 & .00 & .00 & .08 & .10 & .05 & .00 \\
\hline & \multicolumn{8}{|c|}{ Evaluación } \\
\hline & EVAL & .08 & .08 & .00 & .00 & .26 & .18 & .05 \\
\hline & ECOM & .08 & .00 & .00 & .08 & .23 & .15 & .08 \\
\hline
\end{tabular}

Nota:= $\left.P\left(I / C 1, C_{1}\right)\right)=$ probabilidad de presentar el indicador $I$ condicionado a estar en la clase latente $1(C 1)$ y presentar solo una característica $\left(C_{1}\right)$ para el concepto o categoría establecida en el indicador. $\left.P\left(I / C 1, C_{2}\right)\right)=$ probabilidad de presentar el indicador $/$ condicionado a estar en la clase latente $1(C 1)$ y presentar dos características $\left(C_{2}\right)$ para el concepto o categoría establecida en el indicador. $\left.P\left(I / C 1, C_{3}\right)\right)=$ probabilidad de presentar el indicador $I$ condicionado a estar en la clase latente $1(C 1)$ y presentar tres o más características $\left(C_{3}\right)$ para el concepto o categoría establecida en el indicador. $\left.P\left(I / C 2, C_{1}\right)\right)=$ probabilidad de presentar el indicador I condicionado a estar en la clase latente $2(C 2)$ y presentar solo una característica $\left(C_{1}\right)$ para el concepto o categoría establecida en el indicador. $\left.P\left(I / C 2, C_{2}\right)\right)=$ probabilidad de presentar el indicador I condicionado a estar en la clase latente $2(C 2)$ y presentar dos características $\left(C_{2}\right)$ para el concepto o categoría establecida en el indicador. $\left.P\left(I / C 2, C_{3}\right)\right)=$ probabilidad de presentar el indicador I condicionado a estar en la clase latente 2 (C2) y presentar tres o más características $\left(C_{3}\right)$ para el concepto o categoría establecida en el indicador. En negrita las probabilidades condicionales mayores a 4. 
La primera clase latente, denominada DCMM desde el Saber, se conformó por $23 \%$ del total de entrevistados, principalmente por la experiencia de mujeres de entre 60 y 80 años, hablantes de mapudungun de primera lengua y un alto nivel de educación formal. Este grupo se caracteriza por personas que poseen un profundo conocimiento del pueblo, lo que se traduce en altas probabilidades condicionadas con tres características.

Por lo general, este grupo de personas ha desarrollado actividades cotidianas del pueblo en un periodo breve de su niñez basado en la práctica o los relatos de otros, en el contexto de la crianza con abuelas no escolarizadas y hablantes de mapudungun. Por ello, esta clase latente se ha denominado 'Desarrollo de Conocimiento Matemático Mapuche (DCMM) desde el Saber'.

La figura 4 sintetiza la C1 y sustenta el DCMM desde el Saber.

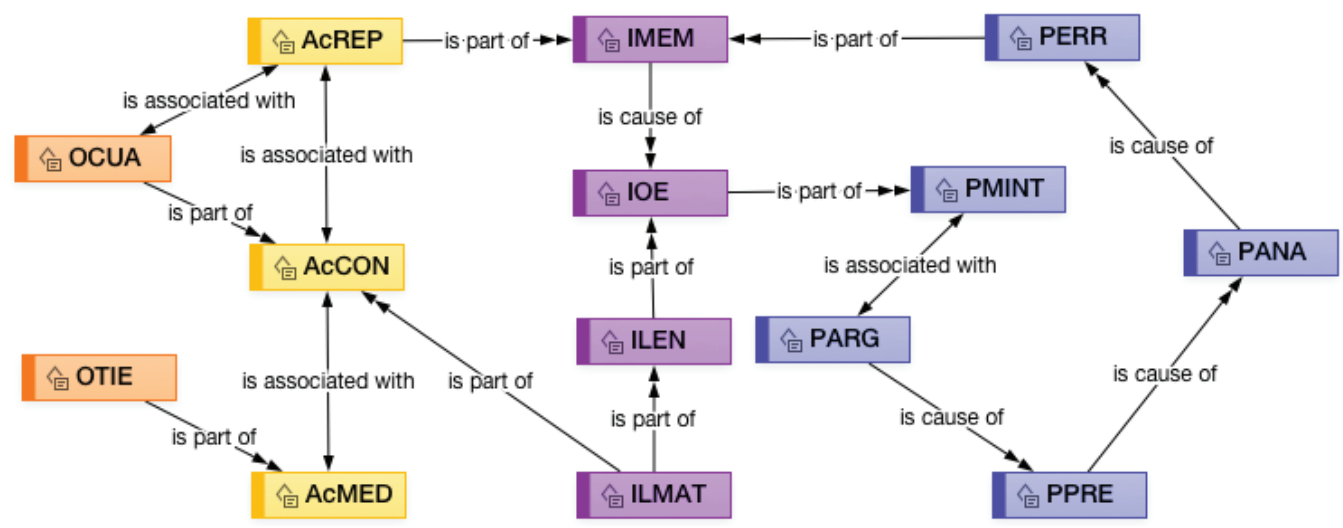

Figura 4. DCMM desde el saber.

El DCMM desde el Saber se sustenta en la memoria colectiva de gente conocedora de procesos y objetos relevantes para el pueblo que, desde la acción o el relato de la acción ( $\mathrm{P}\left(\mathrm{IMEM} / \mathrm{C} 1, \mathrm{C}_{3}\right)=.31 ; \mathrm{P}\left(\mathrm{IOE} / \mathrm{C} 1, \mathrm{C}_{3}\right)=.83$ ), evidencian utilizar lógicas de razonamiento matemático que se acompañan del mapudungun $\left(\mathrm{P}\left(\mathrm{ILEN} / \mathrm{C} 1, \mathrm{C}_{3}\right)=.83 ; \mathrm{P}\left(\mathrm{ILMAT} / \mathrm{C} 1, \mathrm{C}_{3}\right)=.42\right)$ y sus vocablos del sistema numérico, unidades de medidas no estandarizadas, fracciones, operaciones, entre otros.

La C1 se articula bajo el modelamiento de quien posee el conocimiento y se centra en la especificidad del proceso que regula el concepto ( $\mathrm{P}\left(\mathrm{PMINT} / \mathrm{C} 1, \mathrm{C}_{3}\right)=.41$; 
$\mathrm{P}\left(\mathrm{PARG} / \mathrm{C} 1, \mathrm{C}_{3}\right)=$.42; P(PPRE/C1, $\left.\left.\mathrm{C}_{3}\right)=.33\right)$. Así, la acción de medir (P(AcMED/C1, $\left.\left.C_{3}\right)=.25\right)$, contar ( $\left.\left(A C C O N / C 1, C_{3}\right)=.17\right)$ y representar $\left(P\left(A c R E P / C 1, C_{3}\right)=.59\right)$ asociado a los objetos de tiempo (P(OTIE/C1, $\left.\left.\mathrm{C}_{3}\right)=.25\right)$ y cuantificación ( $\mathrm{P}(\mathrm{OCUA} / \mathrm{C} 1$, $\left.C_{3}=.59\right)$ se desarrollan desde la visualización y determinación de patrones de uso, en lógicas contextuales específicas pero flexibles a las demandas del usuario. Cada paso a seguir dentro del proceso y la conceptualización del mismo se nutre y desarrolla a partir del error comparativo $\left(P\left(P E R R / C 1, C_{1}\right)=.25\right)$, esto es, el conocedor del proceso o usuario del objeto matemático evidencia desconformidad en el producto, analiza los conceptos dentro del proceso (P(PANA/C1, $\left.C_{3}\right)=.25$ ) y genera preguntas (P(PPRE/C1, $\left.\mathrm{C}_{3}\right)=.33$ ) y argumentaciones ( $\left(\right.$ PARG/ $\left.\mathrm{C} 1, \mathrm{C}_{3}\right)=.42$ ) que permiten, fortalecer y desarrollar el razonamiento matemático en curso.

La siguiente cita evidencia alguna de estas relaciones:

Yo observaba a mis abuelas, ellas me criaron. No hablaban castellano. Poseían sabiduría asociada a las costumbres del pueblo. (...) ellas salían todos los días con cuatro lanas de diferente color, trenzadas en un extremo y amarradas a su cintura, era para contabilizar sus animales. (...) cuando llegaban a la ruka [casa], comparaban sus registros y sumaban, todos los números en mapudungun y con cálculos mentales o sobre los püron [nudos] (...) lo que más recuerdo eran sus discusiones cuando no coincidían, allí ellas debían hacer memoria y recrear el proceso hasta que ambas quedaban conformes. Fui a la escuela internada, nunca viví el proceso. [E50]

Se observa que desde el cuidado de animales (AANI), el entrevistado recuerda (IMEM) haber escuchado (IOE) la lengua materna, el mapudungun, de sus abuelos (ILEN). De esta manera, el DCMM desde el Saber materializa el desarrollo del conocimiento matemático del pueblo mapuche centrando en el que conoce los procesos-objetos y modela el desarrollo del conocimiento con foco en la memoria oral del mapudungun y el error, el cual propicia la reevaluación, fortalecimiento y re-conceptualización del conocimiento.

Por otra parte, la C2, denominada DCMM desde el Hacer se conforma por $77 \%$ del total de entrevistados, principalmente por un grupo de hombres y mujeres de entre 30 a 60 años del sector rural, hablantes de mapudungun a nivel heterogéneo y educación formal de enseñanza básica o media incompleta monocultural. Esta clase latente se caracteriza por personas reconocidas como referentes expertos dentro de la actividad práctica que desarrollan, aunque entregan escasas características de los conceptos asociados a sus indicadores. Por 
ello, la C2 es denominada 'Desarrollo de Conocimiento Matemático Mapuche (DCMM) desde el Hacer'.

La figura 5 sintetiza la C2 y sustenta el DCMM desde el Hacer.

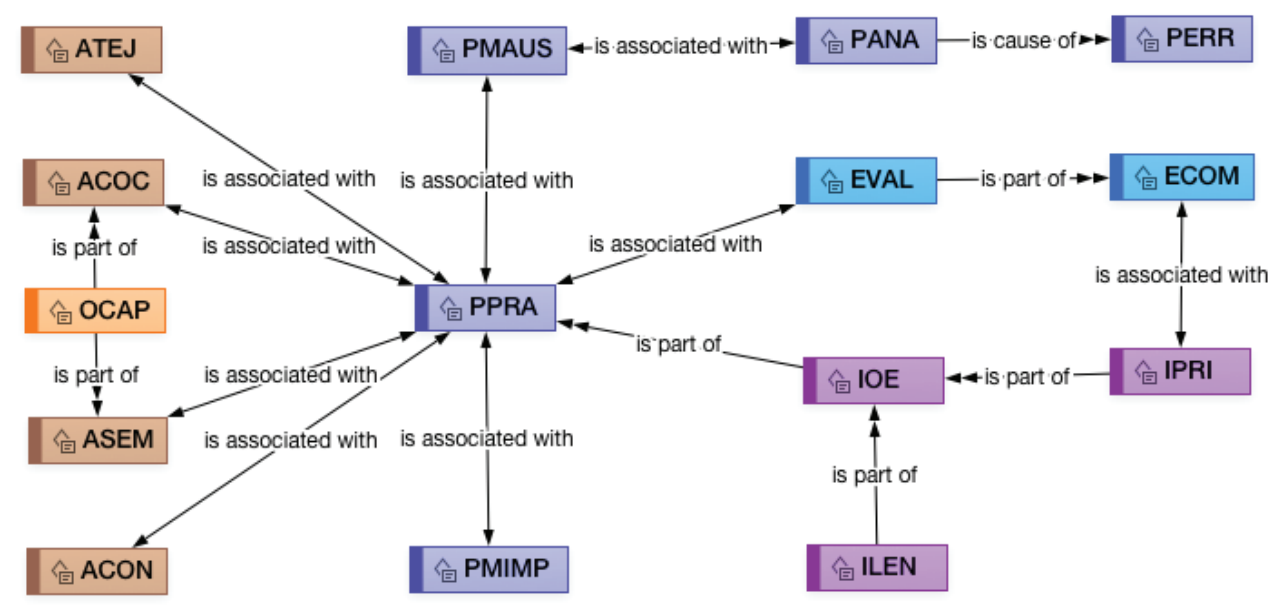

Figura 5. DCMM desde el hacer.

El DCMM desde el Hacer, se sustenta en la notable ejecución de prácticas asociadas a actividades que poseen matemática implícita en su proceso, como lo son tejer $(\mathrm{P}(\mathrm{ATEJ} / \mathrm{C} 2)=.41)$, cocinar $(\mathrm{P}(\mathrm{ACOC} / \mathrm{C} 2)=.15)$, sembrar $(\mathrm{P}(\mathrm{ASEM} / \mathrm{C} 2)=.18)$ y construir $(\mathrm{P}(\mathrm{ACON} / \mathrm{C2})=.26)$, y a objetos físicos que poseen capacidad volumétrica no convencional relacionado principalmente con la siembra y la cocina la cual se debe medir (P(AcMED/C2)=.18) y cuantificar (P(AcCON/C2)=.21). Así, a través de la observación-escucha de la práctica $(\mathrm{P}(\mathrm{IOE} / \mathrm{C} 2)=.90)$, bajo los principios que sustentan su acción y el uso de lexemas de la lengua asociados a artefactos o procesos específicos del mapuche (P(ILEN/C2)=.67), el conocimiento se moviliza desde quien lo porta (P(PMIMP/C2)=.46) imponiendo su conocimiento, hasta quien lo adquiere en un continuo y colaborativo desarrollo desde la acción (P(PMINT/C2)=.46; P(PPRE/C2)=.33).

La movilización del conocimiento se articula desde la imposición de tareas concretas y aisladas dentro de la totalidad de la actividad (P(PMIMP/C2)=.46) o la ausencia del modelador de la práctica en el momento en que se inicie el proceso (P(PMAUS/C2)=.33). Aquí, el análisis del error $(P(P A N A / C 2)=.26)$ junto con la actualización de objetos que intervienen en la actividad fomenta el 
auto-análisis (P(PANA/C2)=.36) y la búsqueda de estrategias personales para evitar confusiones futuras, fortalecen el conocimiento matemático implícito en la actividad, sus lógicas de construcción y uso de artefactos. Finalmente, la evaluación de expertos (P(EVAL/C2)=.26), posibilita la sociabilización de la actividad por medio de la comunicación del producto evaluado $(\mathrm{P}(\mathrm{ECOM} / \mathrm{C} 2)=.26)$. La actividad se conceptualiza en cada fase del proceso, y se fortalece y desarrolla en la comunicación, tal como se observa en las siguientes citas:

Veía a mi mamá tejer a telar (...) ella tejía frazadas, mantas, fajas. Primero me mandaban a ayudar en el lavado de la lana, luego a buscar raíces, para teñir la lana. Me decía qué raíces debía buscar, no sabía por qué, ni qué color daba, inada! Cuando ya era más grande me permitía acompañarla mientras tejía, pero era solo para acercarle cosas, yo no tejía, ni hablaba para no desconcentrarla. [E12]

Aprendí a sembrar solo, mi papá no estaba y mi abuela no podía hacerlo, había que comer así que había que sembrar. No fui más a la escuela y me puse a cultivar la tierra. Mi abuela decía, isiga no más hijo, va a salir bien! La primera siembra me salió toda despareja, pero en la siguiente resolví el problema, solo hice memoria de cómo lo había hecho y pensé una forma para que la semilla no se me amontonara. [E1]

Se observa el modelamiento impuesto (PMIMP) en actividades como el tejido (ATEJ) y la siembra (ASEM), así como la evaluación (EVAL) y el análisis (PANA) de los errores (PERR). De esta manera, el DCMM desde el Hacer materializa el desarrollo del conocimiento matemático del pueblo mapuche centrando en la acción ejecutada la práctica ubicada dentro de un bien comunitario. El desarrollo del conocimiento se modela desde la actualización de insumos para cumplir la tarea, desde donde los procesos antiguos se contrastan con los modernos y se establecen relaciones con matemática implícita en cada una de sus fases.

\section{DISCUSIÓN}

Para establecer el Modelo Condicional/Consecuencial denominado "Desarrollo del Conocimiento Matemático Mapuche (DCMM)", se interpretó lo percibido (Lesh y Harel, 2003) en las entrevistas sobre actividades cotidianas y su forma de transmisión del conocimiento dentro del pueblo mapuche, usando el marco de disposiciones del dominio cognitivo de la competencia profesional del investigador referido al conocimiento matemático (Blömeke et al., 2012) y las redes 
conceptuales/relaciones del modelo de análisis Condicional/Consecuencial (Miles et al., 2014) que surgen a partir del ACL (Magidson y Vermunt, 2004).

El Modelo Condicional/Consecuencial DCMM, es coherente con el desarrollo del conocimiento humano (Rogoff, 2003). Sus clases latentes DCMM desde el Saber y DCMM desde el Hacer logran caracterizar el desarrollo del conocimiento matemático del mapuche, desde la interpretación de saberes y prácticas situadas (Albertí, 2007), en el marco de procesos educativos no formales mapuche en entornos familiares (Alarcón et al., 2018; Peña-Cortés et al., 2019), dependientes del contexto socio-histórico del grupo que lo desarrolla (Quintriqueo et al., 2015).

El análisis descriptivo de esta investigación organiza la totalidad de indicadores emergentes en dos dimensiones denominadas Conocimiento Matemático Mapuche (CMM) y Desarrollo del Conocimiento Mapuche (DCM). Así, el CMM se despliega desde la definición de actividades cotidianas como sembrar, tejer, cocinar, construir y cuidar animales, que contemplan una matemática en principio no observada por la comunidad de forma, dado su cotidianeidad, pero implícita e identificable para un sujeto alfabetizado matemáticamente que tiene intención de diferenciarla. En esta matemática se caracterizan -como ya hemos señalado- dos categorías: acciones y objetos. Las acciones explicitan un conjunto de tareas propias de una práctica matemática, que en cantidad y descripción constituyen un marco estructurado y extenso en componentes conceptuales que integran y amplían en su definición el uso de numerales y cuantificadores en contexto del pueblo mapuche (Catrileo, 1985; Peña-Rincón y Hueitra-Santibañez, 2016; A. Salas, 1980), lo que se estructura en objetos matemáticos.

Por otro lado, el DCM distingue tres marcos de indicadores centrados en el inicio, proceso y evaluación de la transmisión y desarrollo de conocimientos mapuche arraigado en las actividades cotidianas con matemática implícita, pero enfocado en las características y formas en que los actores involucrados despliegan su conocimiento e interactúan con el aprendiz. Este conjunto de indicadores desagrega y profundiza el indicador denominado "Formas de construcción de conocimiento" de Quilaqueo y San Martín (2008), como sus modos de transferencia y enseñanza (Del Pino y Ferrada, 2019; Luna et al., 2018), pero específicas hacia conceptos y objetos matemáticos propios del pueblo mapuche, al estar insertas en el "Saber" y en el "Hacer" de los sujetos entrevistados. Si bien se distinguen tres fases, estas están imbricadas en el desarrollo del CMM, tal como lo plantea Luna et al. (2018) cuando afirman que en los modelos de enseñanza del pueblo mapuche no es posible separar el método 
del conocimiento, ya que este último está inserto en la práctica, siendo a la vez un medio y un fin. Si bien para su comprensión es necesario descomponerlo, tal como ha sucedido con los modelos explicativos del conocimiento del profesor de matemática (Shulman, 1986; Ball et al., 2008; Blömeke et al., 2015), en la práctica estos actúan de forma conjunta. Por lo demás, y dado el modelo de enseñanza, cada familiar o integrante de una comunidad mapuche es a la vez un profesor y aprendiz.

En cuanto al análisis relacional, el CMM evidencia las lógicas de composición entre los objetos y acciones matemáticas interpretadas desde un conjunto finito pero diverso de prácticas culturales mapuche, sin precedentes de investigación. La dimensión CMM como red conceptual no está acabada, posee limitaciones asociadas al número y cualidades de las prácticas culturales de las que emerge (Bishop, 1991). Por otro lado, el DCM como red conceptual, es un proceso amplio en rutas, permeable en contenidos y métodos y cíclico en su progreso, el cual permite entrelazar diversos elementos del pueblo mapuche antes investigados, asociado a lo conceptual, procedimental y valórico-actitudinal que se desprende del formarse como mapuche (Quilaqueo et al., 2016; Quilaqueo y San Martín, 2008). Ambas redes conceptuales permiten tener una base para validar, nutrir y fortalecer desde las investigaciones ya desarrolladas, como también el expandirse y proyectar nuevos estudios.

Finalmente, el análisis Condicional/Consecuencial establece un modelo que caracteriza el DCMM con dos claras rutas de desarrollo de la matemática en el contexto mapuche que coexisten en temporalidad y territorio. El DCMM desde el Saber despliega un desarrollo del conocimiento matemático, rico en objetos, acciones y su conceptualización matemática, modelado por un agente experto en el área que fortalece el proceso explicitando y/o reviviendo los principales puntos de conflicto desde la acción y la oralidad del mapudungun. En cambio, el DCMM desde el Hacer construye una ruta de desarrollo centrado en la práctica cotidiana, donde las acciones y objetos matemáticos son medios implícitos en la tarea. Se aprende haciendo y reflexionando de forma autónoma sobre los errores o nichos de conflicto experimentados. Los conceptos se desarrollan por la actualización de variables que intervienen en la práctica. Así, el Modelo de DCMM corresponde a un formato inédito en su género por dos razones, primero, el comprender el desarrollo del conocimiento matemático dentro del pueblo mapuche ligado a su proceso de enseñanza-aprendizaje y segundo, el comprender el DCMM desde la diferencia socio-histórica del conjunto de personas de 
un sector territorial común, lo que se explicita en cada una de las clases latentes que lo sustentan.

El Modelo de DCMM es una respuesta a los requerimientos establecidos desde la investigación a la enseñanza monocultural de las matemáticas en el sistema escolar chileno (Arias-Ortega et al., 2018; OCDE, 2009; Del Pino y Ferrada, 2019; Luna, et al., 2018; Ortiz-Velosa y Arias-Ortega, 2019). Tanto así, que se puede considerar como una primera aproximación de comprensión del conocimiento y habilidades que un profesor debe tener para enseñar matemática al pueblo mapuche. En él, la familia, la comunidad y sus actividades se establecen como elementos estructurales del conocimiento y de los procesos de enseñanza y aprendizaje en el aula escolar formal (Alarcón et al., 2018). Se identificaron en ellas las acciones y objetos matemáticos propios del pueblo, que configuran, articulan y aseguran la transcendencia del mapuche y su cultura. Sin embargo, se debe reconocer que el Modelo de DCMM como respuesta al modelo monocultural de enseñanza de las matemáticas del sistema escolar, posee limitantes asociadas a la implementación del mismo, por lo que se hace necesario, acompañar el Modelo por un sistema de capacitación profesional docente que posibilite su real implicancia en el sistema escolar.

\section{AGRADECIMIENTOS}

Esta investigación se ha desarrollado dentro del proyecto número 21130915 subvencionado por CONICYT-PFCHA/Doctorado Nacional; y dentro del proyecto número 15110006 subvencionado por CONICYT/FONDAP.

This article was elaborated in the context of INCASI Network, a European project that has received funding from the European Union's Horizon 2020 research and innovation programme under the Marie Sklodowska-Curie GA No 691004 and coordinated by Dr. Pedro López-Roldán. This article reflects only the author's view and the Agency is not responsible for any use that may be made of the information it contains. 


\section{REFERENCIAS}

Alarcón, A., Castro, M., Astudillo, P., y Nahuelcheo, Y. (2018). La paradoja entre cultura y realidad: el esfuerzo de criar niños y niñas mapuche en comunidades indígenas de Chile. Chungará, 50(4), 651-662. https://doi.org/10.4067/S0717-73562018005001601

Albertí, M. (2007). Interpretación matemática situada de una práctica artesanal [Tesis de doctorado no publicada], Universidad Autónoma de Barcelona.

Arias-Ortega, K., Quintriqueo, S., y Valdebenito, V. (2018). Monoculturalism in pedagogical practices along basic teacher education in La Araucanía, Chile. Educaçāo e Pesquisa, 44, 1-19. https://doi.org/10.1590/S1678-4634201711164545

Ball, D. L., Thames M. H., y Phelps, G. (2008). Content Knowledge for Teaching What Makes It Special?. Journal of Teacher Education, 59(5), 389-407. https://doi. org/10.1177/0022487108324554

Barbé, J., Bosch, M., Espinoza, L., y Gascón, J. (2005). Didactic restrictions on the teacher's practice: The case of limits of functions in Spanish high schools. En C. Laborde, M. J. Perrin-Gloria, y A. Sierpinska (Eds.), Beyond the apparent banality of the mathematics classroom (pp. 235-268). Springer-MA. https://doi.org/10.1007/0-387-30451-7_9

Bishop, A. J. (1991). Mathematics enculturation. A cultural perspective on mathematics education. Kluwer Academic Publishers.

Blömeke, S., Gustafsson, J. E., y Shavelson, R. J. (2015). Beyond dichotomies: Competence viewed as a continuum. Zeitschrift Fur Psychologie / Journal of Psychology, 223, 3-13. https://doi.org/10.1027/2151-2604/a000194

Blömeke, S., Hoth, J., Döhrmann, M., Busse, A., Kaiser, G., y König, J. (2015). Teacher change during induction: Development of beginning primary teachers' knowledge, beliefs and performance. International Journal of Science and Mathematics Education, 13(2), 287-308.

Blömeke, S., Suhl, U., Kaiser, G., y Döhrmann, M. (2012). Family background, entry selectivity and opportunities to learn: What matters in primary teacher education? An international comparison of fifteen countries. Teaching and Teacher Education, 28, 44-55. https://doi.org/10.1016/j.tate.2011.08.006

Brousseau, G. (1986). Fundamentos y métodos de la didáctica de las matemáticas. Recherches en Didactique des Mathematiques, 7(2), 33-115.

Canales, A. y Webb, A. (2018). Educational Achievement of Indigenous Students in Chile: School Composition and Peer Effects. Comparative Education Review, 62(2), 231-273.

Catrileo, M. (1985). Concepto y forma de la cuantificación en mapudungun. RLA: Revista de Lingüística Teórica y Aplicada, 23(1), 179-188. 
Chau, K. L. (1990). A model for teaching cross-cultural practice in social work. Journal of Social Work Education, 26(2), 124-133. https://doi.org/10.1080/10437797.1990.10672143 Chevallard, Y., y Bosch, M. (2014). Didactic transposition in mathematics education. En S. Lerman (Ed.), Encyclopedia of mathematics education. Spriger. https://doi. org/10.1007/978-94-007-4978-8_48

D’Ambrosio, U. (2007). La matemática como ciencia de la sociedad. En J. Giménez, J. Díez-Palomar y M. Civil (coords.), Educación Matemática y Exclusión (pp. 83-102), Graó. D’Ambrosio, B. y Lopes, C. (2015). Vertentes da subversão na produção cientifica em educaçāo matemática. Mercado de Letras.

Del Pino, M., y Ferrada, D. (2019). Construction of educational knowledge with the Mapuche community through dialogical-kishu kimkelay ta che research. Educational Action Research, 27(3), 414-434. https://doi.org/10.1080/09650792.2019.1616587

Depaepe, F., y König, J. (2018). General pedagogical knowledge, self-efficacy and instructional practice: Disentangling their relationship in pre-service teacher education. Teaching and Teacher Education, 69, 177-190. https://doi.org/10.1016/j. tate.2017.10.003

Felbrich, A., Kaiser, G., y Schmotz, C. (2012). The cultural dimension of beliefs: an investigation of future primary teachers' epistemological beliefs concerning the nature of mathematics in 15 countries. ZDM - International Journal on Mathematics Education, 44(3), 355-366. https://doi.org/10.1007/s11858-012-0418-x

Ferrare, J. J., y Hora, M. T. (2014). Cultural models of teaching and learning in math and science: Exploring the intersections of culture, cognition, and pedagogical situations. The Journal of Higher Education, 85(6), 792-825. https://doi.org/10.1353/jhe.2014.0030

Finch, W. H., y French, B. F. (2015). Latent variable modeling with R. Routledge.

Flores, J., y Azócar, A. (2006). Fotografía de capuchinos y anglicanos a principios del siglo XX: La escuela como instrumento de cristianización y chilenización. Memoria Americana, 14, 75-87.

Fyhn, A. B., Nutti, Y. J., Nystad, K., Eira, E. J. S., y Hætta, O. E. (2016). We had not dared to do that earlier, but now we see that it works: Creating a culturally responsive mathematics exam. AlterNative: An International Journal of Indigenous Peoples, 12(4), 411-424. https://doi.org/10.20507/AlterNative.2016.12.4.6

Hanisch, W. (1974). Historia de la Compañía de Jesús en Chile (1593-1955). Editorial Francisco de Aguirre, S.A.

Huencho, A. (2015). Estudio de las orientaciones curriculares del Programa Intercultural Bilingüe: un análisis emergente en función de la matemática y la cultura mapuche. Revista Latinoamericana de Etnomatemática, 8(2), 214-236. 
Huencho, A., Rojas, F., y Webb, A. (2017). Educación Matemática Intercultural: Propuestas y proyecciones desde el pueblo mapuche. En E. Treviño, L. Morawietz, C. Villalobos y E. Villalobos (Eds.), Educación intercultural en Chile. Experiencias, pueblos y territorios (pp. 303-334), Ediciones Universidad Católica de Chile.

Ibañez, N. (2010). La atención pedagógica a la diversidad: Estudio en aulas de escuelas rurales de comunidades mapuche del sur de Chile. Educación Superior y Sociedad, 15(2), 83-109.

Kaiser, G., y Maaß, K. (2007). Modelling in lower secondary mathematics classroom problems and opportunities. En W. Blum, P. L. Galbraith, H. W. Henn y M. Niss (Eds.), Modelling and Applicatins in Mathematics Education (pp. 99-108), New ICMI Study Series. https://doi.org/10.1007/978-0-387-29822-1

Kuzniak, A. (2011). L'espace de travail mathématique et ses genèses. Annales de Didactique et de Sciencescognitives, 16, 9-24.

Lesh, R., Sriraman, B., y English, L. (2013). Theories of learning mathematics. En S. Lerman (Ed.), Encyclopedia of Mathematics Education. Springer. https://doi.org/ 10.1007/97894-007-4978-8_157

Lesh, R., y Doerr, H. M. (2003). Foundations of a models and modeling perspective on mathematics teaching, learning, and problem solving. En R. Lesh, y H. M. Doerr (Eds.), Beyond constructivism: Models and modeling perspectives on mathematics problem solving, learning, and teaching (pp. 3-33). Lawrence Erlbaum Associates Publishers. https://doi.org/10.4324/9781410607713

Lesh, R., y Lehrer, R. (2003). Models and modeling perspectives on the development of students and teachers. Mathematical Thinking and Learning, 5(2-3), 109-129. https:// doi.org/10.1080/10986065.2003.9679996

Luna, L., Telechea, C., y Caniguan, N. (2018). Mapuche education and situated learning in a community school in Chile. Intercultural Education, 29(2), 203-217. https://doi. org/10.1080/14675986.2018.1429574

Magidson, J., y Vermunt, J. K. (2004). Latent class models. En D. Kaplan (Ed.), The Sage handbook of quantitative methodology for the social sciences (pp. 175-198), Thousands Oakes, Sage.

Marshall, B., Cardon, P., Poddar, A., y Fontenot, R. (2013). Does sample size matter in qualitative research?: A review of qualitative interviews in is research. Journal of Computer Information Systems, 54(1), 11-22. https://doi.org/10.1080/08874417.2013.11645667

McDonald, M., Kazemi, E., Kelley-Petersen, M., Mikolasy, K., Thompson, J., Valencia, S. W., y Windschitl, M. (2014). Practice makes practice: Learning to teach in teacher education. Peabody Journal of Education, 89(4), 500-515. https://doi.org/10.1080/016195 6X.2014.938997 
Miles, M. B., Huberman, A. M., y Saldaña, J. (2014). Qualitative Data Analysis. A Methods Sourcebook (Third Edit). Sage.

Miller, D. R., Bandura, A., y Walters, R. H. (1966). Social learning and personality development. American Sociological Review, 31(1), 128-130. https://doi.org/10.2307/2091312 Noggler, A. (1982). Cuatrocientos años de misión entre los araucanos. San Francisco.

Ong, W. J. (1987). Oralidad y escritura. Tecnologías de la palabra. Fondo de Cultura Económica.

Organización para la Cooperación y Desarrollo Económicos (OCDE) (2009). La educación superior en Chile. OCDE.

Ortiz-Velosa, E. M., y Arias-Ortega, K. (2019). Being Mapuche in the University: Entry Conditions to the Initial Teacher Training, La Araucanía. Revista Electrónica Educare, 23(1), 1-18.

Peña-Cortés, F., Huiliñir-Curío, V., Pincheira-Ulbrich, J., Quintriqueo, S., Quilaqueo, D., Gutiérrez, M., y Morales, S. (2019). Mapuche-Pewenche knowledge transmitted by teachers and parents: perception of schoolchildren in rural schools of the Araucanía region (Chile). Journal of Multilingual and Multicultural Development, 40(3), 244-256.

Peña-Rincón, P., y Hueitra-Santibañez, Y. (2016). Conocimientos [matemáticos] mapuche desde la perspectiva de los educadores tradicionales de la comuna de El Bosque. Revista Latinoamericana de Etnomatemática, 9, 8-25.

Quilaqueo, D., Quintriqueo, S., y Torres, H. (2016). Características epistémicas de los métodos educativos mapuches. Revista Electrónica de Investigación Educativa, 18(1), 153-165.

Quilaqueo, D., y San Martín, D. (2008). Categorizacion de saberes educativos mapuche mediante la teoría fundamentada. Estudios Pedagógicos XXXIV, 34(2), 151-168. https://doi.org/10.4067/S0718-07052008000200009

Quintriqueo, S. (2010). Implicancias de un modelo Curricular monocultural en contexto mapuche. Universidad Católica de Temuco.

Quintriqueo, S., Quilaqueo, D., Peña-Cortes, F., y Muñoz, G. (2015). Conocimientos culturales como contenido de la educación familiar mapuche. Alpha, 1(40), 131-146. https://doi.org/10.4067/S0718-22012015000100010

Quintriqueo, S., y Torres, H. (2013). Construcción de conocimiento mapuche y su relación con el conocimiento escolar. Estudios Pedagógicos XXXIX, 39(1), 199-216. https:// doi.org/10.4067/S0718-07052013000100012

Restrepo-Ochoa, D.A. (2013). La Teoría Fundamentada como metodología para la integración del análisis procesual y estructural en la investigación de las representaciones sociales. Revista CES Psicología, 6(1), 122-133.

Rogoff, B. (2003). The Cultural Nature of Human Development. Oxford University Press. Rost, J. (2004). Testtheorie-Testkonstruktion [Test theory and test construction]. Verlog Hans Huber. 
Salas, A. (1980). El sistema de numeración en el mapuche de Chile. Revista La Matemática en el Colegio, 4, 30-40.

Salas, S., Godino, J., y Oliveras, M. L. (2015). Números mapuches en el currículo de la lengua mapuzugun en la educación básica chilena. Revista Latinoamericana de Etnomatemática, 8(2), 194-213.

Shulman, L. S. (1986). Those who understand: Knowledge growth in teaching. Educational researcher, 15(2), 4-14.

Shulman, L. S. (1987). Knowledge and teaching. Harvard Educational Review, 57(1), 1-23.

Sierpinska, A., y Lerman, S. (1996). Epistemologies of mathematics and of mathematics education. En A. J. Bishop, K. Clements, C. Keitel, J. Kilpatrick y C. Laborde (Eds.), International Handbook of Mathematics Education (pp. 827-876), HL, Kluwer, A.P.

Strauss, A., y Corbin, J. (2002). Bases de la investigación cualitativa: técnicas y procedimientos para desarrollar la teoría fundamentada. Editorial Universidad de Antioquia.

Turner, F., y Rowland, T. (2011). The knowledge quartet as an organising framework for developing and deepening teachers' mathematics knowledge. En T. Rowland, y K. Ruthven (Eds.), Mathematical knowledge in teaching (vol. 50, pp. 195-212). Springer. https://doi.org/10.1007/978-90-481-9766-8_12

Undurraga, E. (2014). Unraveling development: Three essays on structural determinants of human capabilities [Tesis de doctorado no publicada], Brandeis University.

Van Hiele, P. M. (1959). The child's thought and geometry. En D. Fuys, D. Geddes, y R. Welchman (Eds.). English translations of selected writings of Dina van Hiele-Geldof and Pierre M. van Hiele (pp. 243-252). National Science Foundation.

Verd, J. M., y Lozares, C. (2016). Introducción a la investigación cualitativa. Editorial Síntesis. Vygotsky, L. S. (1978). Mind in society: The development of higher psychological processes. Harvard University Press.

Williamson, G. (2012). Institucionalización de la educación intercultural bilingüe en Chile: notas y observaciones críticas. Perfiles Educativos, XXXIV(138), 126-147.

ANAHÍ HUENCHO

Dirección: Universidad Católica de Temuco

Campus Juan Pablo II

Rudecindo Ortega \#3239, Temuco, Chile.

Edificio Waldo Marchant

Código Postal: 4780000 